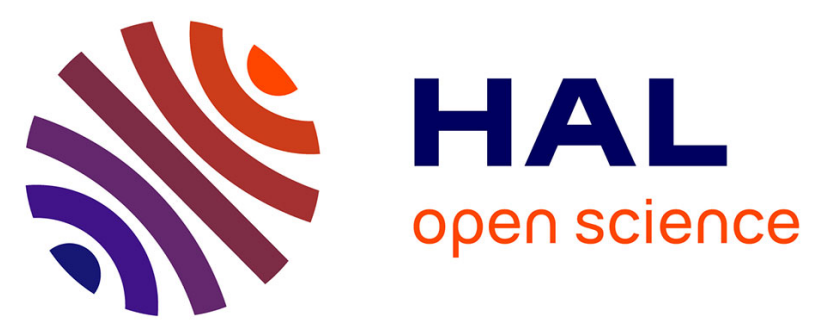

\title{
De la " dynamique " du droit bouddhique birman : D. Christian Lammerts, Buddhist Law in Burma: A History of Dhammasattha Texts and Jurisprudence, 1250-1850, 2018 \\ Grégory Kourilsky
}

\section{To cite this version:}

Grégory Kourilsky. De la " dynamique " du droit bouddhique birman : D. Christian Lammerts, Buddhist Law in Burma: A History of Dhammasattha Texts and Jurisprudence, 1250-1850, 2018. Bulletin de l'Ecole française d'Extrême-Orient, 2018, 104 (1), pp.377-385. 10.3406/befeo.2018.6279 . halshs-02866542

\author{
HAL Id: halshs-02866542 \\ https://shs.hal.science/halshs-02866542
}

Submitted on 12 Jun 2020

HAL is a multi-disciplinary open access archive for the deposit and dissemination of scientific research documents, whether they are published or not. The documents may come from teaching and research institutions in France or abroad, or from public or private research centers.
L'archive ouverte pluridisciplinaire HAL, est destinée au dépôt et à la diffusion de documents scientifiques de niveau recherche, publiés ou non, émanant des établissements d'enseignement et de recherche français ou étrangers, des laboratoires publics ou privés. 
De la « dynamique » du droit bouddhique birman : D. Christian Lammerts, Buddhist Law in Burma: A History of Dhammasattha Texts and Jurisprudence, 1250-1850, 2018

\section{Gregory Kourilsky}

\section{Citer ce document / Cite this document :}

Kourilsky Gregory. De la « dynamique » du droit bouddhique birman : D. Christian Lammerts, Buddhist Law in Burma: A History of Dhammasattha Texts and Jurisprudence, 1250-1850, 2018. In: Bulletin de l'Ecole française d'Extrême-Orient. Tome 104, 2018. pp. 377-385;

doi : https://doi.org/10.3406/befeo.2018.6279

https://www.persee.fr/doc/befeo_0336-1519_2018_num_104_1_6279

Fichier pdf généré le 07/01/2020 


\title{
De la «dynamique» du droit bouddhique birman
}

\author{
Gregory KOURILSKY*
}

D. Christian LammerTs, Buddhist Law in Burma: A History of Dhammasattha Texts and Jurisprudence, 1250-1850, Honolulu, University of Hawai' $\mathrm{i}$ Press, 2018, xiv + 288 pages, appendice, bibliographie, index - ISBN 978$0-8248-7260-1 ; 65 \$$

Le «droit bouddhique» de Birmanie a très tôt suscité l'intérêt des orientalistes. Dès le milieu du XIX ${ }^{\mathrm{e}}$ siècle, un certain nombre de Dhammasat (sanskrit Dharmaśāstra, pali Dhammasattha), codes juridiques rédigés en birman ou en pali et qui constituent un genre particulier dans la littérature bouddhique locale, ont fait l'objet d'études et de traductions de la part de missionnaires et d'administrateurs des Indes britanniques dont il faut saluer les études pionnières - celles de David Richardson ${ }^{1}$, John Jardine ${ }^{2}$, Alois Anton Führer ${ }^{3}$, Emmanuel Forchhammer ${ }^{4}$. Ces études se sont toutefois limitées à un type de textes particulier, en l'occurrence ceux qui faisaient référence à Manu, le législateur primordial de la mythologie indienne, car ils permettaient d'inscrire la littérature juridique birmane dans la tradition des Dharmaśāstra dont l'ancienneté est estimée aux premiers siècles de l'ère chrétienne ${ }^{5}$. Des hypothèses furent alors formulées pour expliquer dans quelles circonstances des traités de tradition brahmanique, rédigés en sanskrit, avaient abouti à des codes de loi civile, rédigés en langues vernaculaires (birmane en premier lieu mais aussi môn, arakanaise, thaïe ou lao) et parsemés de termes et de passages en pali empruntés au corpus canonique ou paracanonique du bouddhisme du Theravāda. Considérant le début prometteur de ces études, on s'étonne qu'elles aient, par la suite, si peu inspiré le milieu académique: mis à part une poignée de travaux publiés au cours du siècle suivant (en particulier par Robert Lingat et Andrew

\footnotetext{
* Chercheur associé à l'École française d'Extrême-Orient, gregory.kourilsky@efeo.net.

1. David Richardson, The Dhamathat, or The Laws of Menoo, Moulmein, American Baptist Mission Press, 1847.

2. John Jardine, Notes on Buddhist Law, 8 tomes, Rangoun, Government Printing Office, 1882-1883.

3. Alois A. Führer, «Manusāradhammasațtham, the Only One Existing Buddhist Law Book, Compared to the Brahmanical Mānavadharmaśāstram », Journal of the Royal Asiatic Society, 15, 1883, p. 329-338.

4. Emmanuel Forchhammer, The Jardine Prize: An Essay on the Sources and Development of Burmese Law, Rangoun, Government Printing Office, 1885. King Wagaru's Manu Dhammasattham. Text, translation and notes, Rangoun, Government Printing, 1892.

5. Une notable exception est l'édition et la traduction établies par E. Forchhammer et Moung Theka Phyoo du Mohavicchedanī Dhammathat (dans J. Jardine, Notes on Buddhist Law, op. cit., tome 6, p. 1-7), dont il sera question ci-après.
} 
Huxley), les codes juridiques birmans et, plus largement, ceux de l'Asie du Sud-Est continentale, ont été laissés dans l'ombre des bibliothèques et des monastères.

Reprenant le fil d'une recherche prometteuse mais délaissée depuis plus d'un siècle, l'ouvrage de D. Christian Lammerts, Buddhist Law in Burma: A History of Dhammasattha Texts and Jurisprudence, 1250-1850, suscitera l'intérêt des spécialistes des traditions religieuses de l'Asie du Sud-Est mais aussi celui des chercheurs, de plus en plus nombreux, s'intéressant au «droit bouddhique», c'est-à-dire aux questions d'ordre juridique qui traversent les communautés bouddhistes au-delà du code monastique ou Vinaya. Buddhist Law in Burma remplit deux objectifs principaux: retracer une généalogie des Dhammasattha birmans à partir de l'analyse d'un choix de textes particulièrement significatifs et tenter de définir la place qu'ils occupent dans le bouddhisme local; esquisser les contours d'une histoire du droit birman jusqu'au début de la période coloniale pour mettre en lumière les enjeux politiques et sociaux sous-jacents à leur genèse, et leur évolution.

Le corps de l'ouvrage est divisé en deux parties traitant de problématiques distinctes mais qui n'en obéissent pas moins à la chronologie historique. La première («Sources») passe en revue les sources anciennes (inscriptions et manuscrits principalement) afin de reconstituer une histoire des Dhammasattha entre le XIII ${ }^{\mathrm{e}}$ et la première moitié du XVII ${ }^{\mathrm{e}}$ siècle; une place importante est accordée à deux textes emblématiques de cette période, le Dhammavilāsa-dhammasat et le Manusāra-dhammasattha, le premier étant le plus ancien du genre qui nous soit parvenu (p. 46). La seconde partie («Revisions and reasons») considère le tournant significatif qu'a pris en Birmanie le regard porté sur les Dhammasat à partir de la fin du XVII ${ }^{e}$ siècle, lorsque des entreprises de réforme religieuse aboutirent à leur réexamen à la lumière des écrits canoniques du bouddhisme theravāda (p. Tipitaka, birm. Pițakat) avec lesquels ils n'étaient pas toujours en conformité, pouvant même, sur certains points, présenter des vues opposées. Cette période donna lieu à un renouvellement du genre des Dhammasattha qui se prolongea jusqu'après l'intégration de la Birmanie à l'Empire britannique. Cette seconde partie de l'ouvrage vise également à analyser les causes politiques, religieuses et sociales qui ont permis et favorisé ce développement.

Le lecteur de Buddhist Law in Burma sera frappé par l'utilisation très abondante des sources primaires, dont beaucoup sont inédites. Cette démarche doit être d'autant plus soulignée que les études contemporaines sur l'Asie du Sud-Est continentale ont parfois tendance à négliger ces sources qui sont, il est vrai, d'accès difficile puisqu'il faut en maîtriser les différents idiomes (ceux des populations locales mais aussi les langues «classiques» du bouddhisme, le sanskrit et le pali) ainsi que les multiples graphies dans lesquelles elles sont consignées. Leur examen nécessite ensuite une bonne connaissance des traditions autochtones, à laquelle doit s'ajouter celle des conceptions et des écrits du bouddhisme canonique, sur lesquels celles-ci s'appuient avec plus ou moins de fidélité. D. C. Lammerts s'acquitte ici parfaitement de sa tâche puisqu'il présente, 
tout au long de l'ouvrage, une multitude d'extraits de Dhammasat et d'autres écrits soigneusement choisis pour illustrer son sujet, édités, traduits de première main (du birman, du pali et même de l'arakanais) et savamment commentés. Une quarantaine de textes sont ainsi étudiés, dont beaucoup à partir de multiples copies manuscrites. Quelques-uns de ces textes font l'objet d'une analyse détaillée (en particulier le Dhammavilāsadhammasat et le Manusāra-dhammasattha évoqués précédemment, mais aussi des textes plus tardifs tels que le Mahäbuddhankkura-dhammasat, le Vinicchayabhedaka-dhammasat ou le Manu kyay - inédits en langues occidentales, à l'exception du dernier). Le tour de force de C. Lammerts est certainement d'avoir réussi à dépasser le stade de la simple analyse textuelle (ce qui aurait déjà été suffisant pour considérer son travail comme digne d'intérêt) en articulant l'ensemble des sources qu'il étudie dans une perspective historique et épistémologique, qu'il qualifie lui-même de dynamic jurisprudence.

Il faut s'arrêter un instant sur ce dernier terme («jurisprudence») que C. Lammerts emploie de façon constante sans jamais le définir précisément, ce qui peut être perturbant pour le lecteur francophone. Dans la langue française moderne, en effet, la jurisprudence renvoie à un ensemble de jugements ou de décisions prises par diverses instances judiciaires qualifiées pour donner une interprétation de la loi. Dans la langue anglaise, ce terme a une acception plus large. Il peut désigner la science ou la philosophie du droit ainsi que l'étude des lois et des principes sur lesquels elles sont basées ${ }^{6}$. L'auteur de Buddhist Law in Burma emploie quant à lui le terme «jurisprudence» dans ces deux acceptions, sans toujours les dissocier.

Dans la présentation que donne C. Lammerts des Dhammasat birmans et de leur évolution au cours des siècles, on ne sera pas étonné que les données relatives aux périodes récentes (XVIII-XIX ${ }^{\mathrm{e}}$ siècles) soient plus fournies que celles concernant les périodes plus anciennes. En effet, les documents relatifs aux Dhammasattha antérieurs au XVII ${ }^{\mathrm{e}}$ siècle sont, pour l'essentiel, de nature épigraphique (p. 43). La part consacrée à la période 1250-1600 est donc assez brève ( 25 pages) et porte davantage sur la mention du ou des (?) Dhammasattha dans les inscriptions et sur ce qu'il est possible d'en déduire, que sur leur contenu concernant lequel ces témoignages restent laconiques. La littérature birmane livre néanmoins certaines informations d'ordre juridique relatives à cette période, telle que la transcription manuscrite de décisions de justice (p. vinicchaya) rendues vers 1610 par un moine nommé Sirīsangghapāla, se rapportant à l'habillement du moine et au droit pour les religieux de disposer de serviteurs (ou d'«esclaves») (p. 37-43).

Ce n'est qu'à partir du milieu du XvII ${ }^{\mathrm{e}}$ siècle qu'il est possible de se faire une idée assez précise de la nature des Dhammasat birmans, en particulier du Dhammavilāsa-dhammasat et du Manusāra-dhammasattha dont les dates de composition sont connues (respectivement 1637-1638 et 1651-1652),

6. La langue française reconnaissait, jusqu'au XIX ${ }^{\mathrm{e}}$ siècle, cette double acception. Ce n'est plus le cas aujourd'hui. 
bien que les copies manuscrites dont on dispose soient sensiblement plus tardives. Contemporains et relevant du même genre littéraire, les deux textes présentent de nombreux points communs, partagés semble-t-il par l'ensemble des Dhammasattha de Birmanie: une structure articulée en dixhuit points ou chapitres correspondant aux différents types de contentieux (sk. vyavahāra) définis dans les Dharmaśāstra sanskrits (p. 59) - relatifs à la dette, au dépôt, à la propriété foncière, etc. Ces textes, bien qu'ils soient rédigés en langue vernaculaire, s'appuient abondamment sur le corpus pali, par un recours à des notions, des références et des citations issues des écrits canoniques et paracanoniques (le Vinaya en premier lieu, mais pas seulement). La première partie du Manusāra-dhammasattha, intitulée Manusāra-pātha, est même entièrement rédigée en pali, la seconde partie en étant la glose (nissaya) en birman, quoique celle-ci contienne des développements absents de son modèle supposé (p. 94). Cette asymétrie éclaire le fait que la relation entre les textes pali et ceux composés en langues vernaculaires n'est pas univoque dans les traditions bouddhiques sud-asiatiques, les premiers n'étant pas toujours générateurs des seconds. Le recours systématique au Vinaya, à ses commentaires et para-commentaires (atthakathā, mūlațīk $\bar{a}$, anuțīka $)$ ne doit pas conduire à assimiler les Dhammasat à des codes monastiques (p. 6). S'ils concernent pour partie les moines, ces textes ressortissent dans leur ensemble à une législation civile, étant entendu que dans les «royautés bouddhiques», la population du monastère n'est pas retranchée du monde mais incarne un corps social de premier plan, en interaction constante avec les autres. Le caractère séculaire des Dhammasat a imposé à leurs rédacteurs de revisiter parfois, voire de détourner, le Tipitaka pour élargir le domaine d'application des lois monastiques à l'ensemble de la société comme, par exemple, pour statuer sur la destination des biens d'un bonze décédé (p. 75, 87). Le hiatus avec certaines règles établies dans le Vinaya conduit d'ailleurs, à partir de la seconde moitié du $\mathrm{XVII}^{\mathrm{e}}$ siècle, les élites religieuses et les juristes birmans à entreprendre une relecture critique des Dhammasattha en les confrontant avec les sources pali, ce qui aura pour effet collatéral de délégitimer, dans une certaine mesure, les codes juridiques traditionnels (p. 88). Ainsi le clerc birman Vaṇnadhamma, dont au moins trois textes nous sont parvenus, indique-t-il dans le Vinicchayapakāsinī (1771) avoir corrigé et «purifié» les articles de loi des anciens Dhammasat lorsqu'ils se trouvaient en contradiction avec les sources canoniques ( $p \bar{a} l \grave{l})$ (p. 167). À ce sujet, il est surprenant que C. Lammerts ne fasse pas mention du Mohavicchedanī Dhammathat, code birman portant sur les droits de succession inspiré formellement, comme son titre l'indique, du commentaire sur les mâtikā ou «tables des matières» des sept livres de l'Abhidhamma attribué à Kassapatthera, moine bouddhiste indien ayant vécu à la fin de la dynastie des Cōḷa (i.e. XII-XIII ${ }^{\mathrm{e}}$ siècles). Ce texte tardif (1832) évacue de façon significative toute référence à Manu et fait du Buddha le premier législateur ${ }^{7}$. Les Pitakat samuinh (catalogues ou inventaires des textes bouddhiques connus localement au moment de

7. $C f$. note 5 . 
leur élaboration) des XVIII et XIX ${ }^{\mathrm{e}}$ siècles poursuivent cet examen critique, jusqu'à affirmer que les Dhammasat ne relèvent pas de l'«Enseignement [du Buddha]» (sâsana) mais d'une littérature se rapportant à la sphère mondaine (lokiya) et que, de ce fait, ils ne doivent pas être considérés comme faisant partie du Pitakat (p. 140-143). Par voie de conséquence, la tradition juridique birmane aboutit à établir une séparation claire entre les juridictions monastique et séculaire (p. 90) et à attribuer «deux corps légaux» (two legal bodies) au bonze, celui-ci devant être placé à la fois sous l'autorité supramondaine du Vinaya et sous celle de la loi mondaine définie par les Dhammasattha (p. 43).

Ces considérations sont fondamentales pour l'histoire des idées en Asie du Sud-Est (et sans doute au-delà) tant il est tenu pour acquis que l'examen critique des textes et des pratiques religieuses, en particulier lorsqu'il s'agit de questionner leur validité à la lumière des écrits primitifs ou plus anciens, ne serait apparu localement qu'à la période coloniale, autrement dit qu'une telle démarche intellectuelle serait le résultat d'une influence exercée par les orientalistes européens. Outre ce qui concerne des bouleversements éclatants (par exemple l'abandon relatif de l'hindouisme au profit du bouddhisme au Cambodge vers la fin de la période angkorienne), les écrits académiques ont en effet tendance à donner de la vie religieuse de la péninsule Indochinoise pré-coloniale une image relativement figée, avec des traditions immunes à tout changement et des textes sans genèse ni auteurs. En choisissant d'une part d'adopter une approche émique, d'autre part de proposer une mise en perspective diachronique, D. C. Lammerts fait sans conteste preuve d'innovation dans son examen $\mathrm{du}$ «droit bouddhique» birman.

La question se pose ensuite de savoir pourquoi les juristes, au tournant $\mathrm{du} \mathrm{XVIII}^{\mathrm{e}}$ siècle, ont cru devoir reconsidérer une législation pluriséculaire. L'auteur de Buddhist Law in Burma ne répond pas vraiment à cette question, sauf à replacer cette évolution dans un mouvement plus large de «purification» religieuse qui conduisit au réexamen de tout le corpus bouddhique birman à l'aune des textes canoniques (p. 174). Sans doute l'état des connaissances de l'histoire birmane est-elle encore trop embryonnaire pour apporter une réponse précise à cette interrogation. Il est probable que ces entreprises répondirent à des changements sociopolitiques profonds ayant marqué la Birmanie à cette époque - de la même façon que des théories juridiques nouvelles se sont imposées en Europe aux $\mathrm{XVI}^{\mathrm{e}}$ et $\mathrm{XVII}{ }^{\mathrm{e}}$ siècles (celles par exemple de Jean Bodin en France ou de John Locke en Angleterre) dans un contexte de crises politiques et de réformes religieuses. C. Lammerts met d'ailleurs en évidence le fait que les Dhammasat birmans étaient d'abord destinés aux juges (nous avons donc bien affaire à un droit positif) et non aux moines (p. 182) - bien que ces deux fonctions aient pu éventuellement coïncider - et surtout qu'ils étaient systématiquement rédigés sous le patronage royal, si ce n'est initiés par les princes eux-mêmes (p. 173). Il est en tout cas permis d'envisager une alternative à l'interprétation «moderniste» proposée par l'auteur - sans l'écarter pour autant - qui voit dans ces réformes le signe d'une mutation historique irréversible, en substance un changement de paradigme corollaire du basculement de la Birmanie vers la modernité. 
En réalité, de telles entreprises de rénovation du bouddhisme pour revenir à une orthodoxie réelle ou supposée, loin d'être spécifiques à la Birmanie $\mathrm{du} \mathrm{XVII}^{\mathrm{e}}$ siècle, sont constantes au fil de l'histoire religieuse régionale à Pagan, au Lan Na, au Siam, au Sri Lanka, où elles prirent la forme de disputes normatives, d'organisation de conciles (p. sañgayāna) ou encore de refontes du corpus. Pareils cycles de réformes invitent à relativiser la vision wébérienne d'un affrontement univoque entre modernité et tradition. Ils doivent conduire à envisager, au contraire, la persistance dans l'histoire du bouddhisme theravāda d'une tension structurelle qui implique que les pratiques religieuses sont régulièrement discutées ou remises en cause, de façon plus ou moins explicite, au gré des contingences socio-historiques. Bien que le nombre de textes examinés par C. Lammerts soit considérable, seule l'étude exhaustive du genre des Dhammasat pourra nous dire si cette dynamique critique est réellement décisive ou, au contraire, si elle participe d'un processus régulier dans l'histoire et la sociologie du bouddhisme de l'Asie méridionale et sud-orientale. Il est remarquable, à cet égard, que cette dynamique de "purification» n'ait pas remis en cause la légitimité des Dhammasat, qui continuèrent d'être produits, diffusés et utilisés en Birmanie jusqu'au XIX ${ }^{\mathrm{e}}$ siècle avant d'être remplacés, sous la pression des autorités coloniales britanniques, par des textes d'une autre nature, les Upade (p. upadesa) (p. 178-180).

La question majeure qui traverse le livre Buddhist Law in Burma est celle de la nature et de l'origine des Dhammasat birmans. Bien que leurs auteurs se soient largement appuyés sur le corpus pali, en particulier le Vinaya et les écrits qui en sont dérivés - même s'ils peuvent s'en écarter ou les contredire -, les Dhammasattha ont également puisé, de manière directe ou indirecte, à d'autres sources, en premier lieu les Dharmaśāstra de l'Inde brahmanique (smrti). Rédigés en sanskrit versifié et remontant probablement, pour les plus anciens, aux premiers siècles de l'ère chrétienne, les Dharmaśāstra (dérivés eux-mêmes des Dharmasūtra plus anciens) sont, comme leur nom l'indique, des «traités de Loi», étant entendu que ce dernier terme (sk. dharma, p. dhamma, «loi») doit ici être compris dans ses deux dimensions, juridique et cosmique. Les Lois de Manu (Mānavadharmaśāstra ou Manusmrti) est, dans ce corpus, le document le plus célèbre. L'emprunt le plus évident des Dhammasattha aux Dharmaśāstra est leur articulation systématique en dix-huit catégories de litiges (p. mūla, litt. «racine»), mentionnées ci-dessus, qui répondent aux vyavahārapada énumérés, en nombre égal, dans les traités indiens. D'autres schèmes leur font par ailleurs écho, tel le recours récurrent à des nomenclatures ou des classifications thématiques reconnaissant par exemple «vingt-cinq types de vol» (pañcavisati avahāra) ou «seize catégories de fils», bien que quelquesuns de ces schèmes se trouvent également, dans une forme parfois modifiée, dans les textes pali (p. 73-74, 96).

Comme cela est signalé dans l'introduction, certains observateurs, notamment Max Weber et Thomas W. Rhys-Davids, ont fait remarquer que le bouddhisme ancien n'avait pas constitué de véritable droit en dehors du code monastique (Vinaya), dans la mesure où le Buddha et ses disciples 
entendaient vivre en marge de la société et recréer un ordre autonome régi par ses propres règles. Cependant, le bouddhisme déborda rapidement la communauté des moines et les adeptes laïcs, par nature non assujettis aux lois monastiques, continuaient par la force des choses d'être soumis - c'est la thèse de Robert Lingat - au «droit brahmanique » ${ }^{8}$. Aussi les Dharmaśāstra n'étaient-ils pas circonscrits aux populations d'obédience brahmanique et la société civile indienne n'imposait-elle pas de séparation stricte entre bouddhistes et hindouistes; au niveau légal, tous étaient placés sous la même juridiction. Le fait est que ni l'Inde ni le Sri Lanka ne semblent avoir produit de version ou d'adaptation pali des Dharmaśāstra. Les Dhammasattha n'existent donc, jusqu'à plus ample informé, qu'en Asie du Sud-Est continentale. La thèse communément admise est que ce sont les Môn qui auraient produit les premiers Dhammasattha, qu'ils auraient dans un premier temps rédigés en pali à partir d'un ou plusieurs Dharmaśāstra sanskrits tout en leur donnant une forme bouddhique9. Le genre des Dhammasat se serait développé par la suite en Asie du Sud-Est à partir de cette matrice écrite en pali, d'abord en môn, puis en birman, avant de suivre une évolution singulière dans le monde thaï-lao. Tout en reconnaissant une parenté certaine entre les Dhammasat môn-birmans et les Dharmaśāstra de l'Inde brahmanique, D. C. Lammerts s'inscrit en faux contre l'idée d'une parenté directe entre les deux corpus - idée résultant selon lui d'une surévaluation de l'importance du Manu-dhammasattham (ou «Le Dhammasat du roi Wagaru»), adaptation môn des Lois de Manu, dont une recension birmane fut traduite et éditée par E. Forchhammer $(1892)^{10}$ et servit de base aux autorités britanniques pour leur refonte du droit birman (p. 13-17). Il pense plutôt que leurs similitudes provient d'un socle commun aux traditions légales sanskrites du sous-continent indien, par-delà les étiquettes religieuses (p. 87). Il accrédite ici la possibilité, jadis avancée par Forchhammer, de l'existence en Inde même d'un Dharmaśāstra bouddhique, aujourd'hui disparu. Ce point est peut-être le seul de tout l'ouvrage sur lequel nous garderons une certaine réserve. Non que la proposition de C. Lammerts ne soit pas vraisemblable - elle l'est sans aucun doute - mais parce qu'il est permis d'estimer que ce dernier évacue un peu rapidement l'hypothèse, formulée par plusieurs de ces prédécesseurs, d'une filiation relativement directe entre Dharmaśāstra (brahmaniques) et Dhammasat. Il fournit d'ailleurs lui-même des arguments a contrario de la thèse qu'il avance en donnant de nombreux exemples de similitudes entre les traités hindous et les codes birmans évoqués précédemment. En outre, il relève la présence de nombreuses occurrences de l'expression sanskrite «dharmaśāstra» dans l'épigraphie de l'Asie du Sud-Est qui permettent d'affirmer que ces textes étaient connus au moins parmi certaines communautés brahmaniques, mais aussi bouddhiques, de la région entre le premier millénaire et la première

8. Robert Lingat, «La conception du droit dans l'Indochine hînayâniste», Bulletin de l'École française d'Extrême-Orient, 44 (1), p. 163-187.

9. Cf. R. Lingat, op. cit., p. 168.

10. E. Forchhammer, King Wagaru's Manu Dhammasattham ... op. cit. 
moitié du second. Il rappelle également, avec justesse, la circulation dans la région de traités politiques (rājaśāstra), horoscopiques (horaśāstra) et grammaticaux issus de la tradition sanskrite ou directement influencés par elle, bien que les versions indochinoises connues soient rédigées dans les langues locales. Encore faut-il ajouter que la civilisation angkorienne, dont la langue savante était exclusivement le sanskrit, a marqué des zones aussi septentrionales que Sukhothaï (Thaïlande), en contact avec la BasseBirmanie dès la première moitié du second millénaire. Encore plus loin vers le nord, les inscriptions môn de la région de Lamphun (Thaïlande) - l'un des épicentres du bouddhisme du Theravāda en Asie du Sud-Est - témoignent encore de l'influence que la culture sanskrite a pu exercer dans cette zone géographique.

S'il est vrai que les sources connues ne permettent pas d'envisager la présence dans la Birmanie pré-coloniale de communautés ou de mouvements religieux se réclamant strictement du brahmanisme ou même du bouddhisme du Mahāyāna, il n'en demeure pas moins que des textes sanskrits appartenant à ces courants y ont bien circulé, même s'ils ont été rapidement absorbés par le bouddhisme de langue pali. Certes, ces éléments ne constituent pas une preuve irréfutable de la présence de Dharmaśāstra à Pagan ou à Pégu à une époque ancienne - les exemplaires introduits tardivement à partir du Bengale ne sont, quant à eux, guère significatifs (p. 154) - mais ils forment à tout le moins une somme d'indices non négligeables. Quant à l'argument avancé par C. Lammerts, selon lequel les témoignages relatifs aux Dhammasattha suggèrent que ceux-ci étaient, dès le $\mathrm{XvI}^{\mathrm{e}}$ siècle, rédigés en birman (p. 90-91), il ne convainc pas davantage puisqu'il n'interdit nullement la possibilité d'une rédaction plus ancienne (qui pourrait être attribuée aux Môn, ou même aux Pyu, dont les civilisations sont attestées dès les premiers siècles du premier millénaire), à partir de laquelle des traductions ou adaptations en birman auraient été élaborées plus tardivement.

En tout état de cause, que le lecteur soit convaincu ou non par l'hypothèse privilégiée par C. Lammerts, le plus important est que ce dernier offre sur le sujet de la genèse des Dhammasat birmans des pistes de réflexion aussi riches que stimulantes.

L'ouvrage est rédigé dans une écriture à la fois élégante et précise, rendant la lecture agréable et le propos lumineux. La présence, en fin de volume, d'un très riche appareillage de notes, d'un index et surtout d'une annexe reprenant pas moins de quatre inventaires de Dhammasattha établis par des lettrés birmans entre 1768 et 1818 (dont la simple existence témoigne de la «dynamique critique» qui traverse l'histoire du droit bouddhique birman) viennent compléter l'ensemble. On regrettera néanmoins l'absence d'une carte de la Birmanie et de l'Asie du Sud-Est péninsulaire, qui reflète la faible part accordée à la géographie dans cet ouvrage. La mise en perspective de l'étendue de l'espace birman dans la péninsule Indochinoise occidentale et de sa position par rapport aux ensembles civilisationnels voisins aurait sans doute permis de mettre en évidence, et peut-être d'expliquer en partie, l'existence de deux traditions juridiques distinctes dans cette région unifiée culturellement par le bouddhisme de langue pali (le Pali imaginaire défini 
par Steven Collins ${ }^{11}$ ) depuis au moins le XIV siècle: d'une part celle du pays môn, de la Birmanie, du Lan Na et du Laos, qui s'appuie de façon explicite sur le corpus du bouddhisme theravāda; de l'autre, celle d'Ayutthaya et du Cambodge, davantage marquée du sceau du brahmanisme quoique dépouillée des éléments structurels qui fondent les sociétés indiennes. Seule la première peut être qualifiée, le cas échéant, de «droit bouddhique» (Buddhist law).

L'approche philologique et diachronique par laquelle D. Christian Lammerts a choisi d'étudier les Dhammasat birmans est des plus pertinentes, d'autant que son entreprise est menée avec une rigueur et une érudition incontestables. Par la nature du sujet traité, par la qualité des analyses proposées et la richesse des sources exploitées, Buddhist Law in Burma fera date et s'inscrit d'ores et déjà dans la liste des ouvrages de référence sur le bouddhisme de l'Asie du Sud-Est.

11. Steven Collins, Nirvana and Other Buddhist Felicities. Utopias of the Pali imaginaire, Cambridge, Cambridge University Press, 1998. 